\title{
MedienPädagogik
}

Zeitschrift für Theorie und Praxis der Medienbildung

\section{Qualitative Forschung auf der Basis von Eigenproduktionen mit Medien.}

\section{Erfahrungswerte aus dem EU-Forschungsprojekt CHICAM - Children In Communication About Migration}

Horst Niesyto und Peter Holzwarth

\section{Einleitung}

Visuelle Methoden haben in verschiedenen Bereichen qualitativer Forschung eine wichtige Bedeutung. Zu nennen sind vor allem die visuelle Soziologie und die visuelle Anthropologie. Fotografie und Video werden bei teilnehmender Beobachtung zusätzlich zu Feldnotizen eingesetzt. Video dient zur Dokumentation von Interviews und Gruppendiskussionen. Bilder oder Filmsequenzen sind geeignet, um Kommunikation im Rahmen von Interviews zu stimulieren („photo-elicitation“, vgl. Prosser/Schwartz 1998, S. 123). In Pierre Bourdieus Arbeiten lassen sich einige interessante Beispiele für diesen Ansatz finden (Bourdieu 1987, S. 87). Eine weitere Möglichkeit besteht darin, bereits existierende visuelle Darstellungen von Subjekten zum Gegenstand der Analyse zu machen (z.B. Kinderzeichnungen oder Graffiti-Malereien; vgl. Neuß 1999; Holzwarth 2001). Interessante Erfahrungswerte gibt es auch im umfangreichen Gebiet des ethnologischen Films (u.a. Curtis, Flaherty, Mead, Rouch), insbesondere das dialogische Vorgehen bei Rouch (die Kamera als integraler Bestandteil der Erfahrung und Erkenntnis sozialer Wirklichkeit; vgl. Friedrich 1984).

Teilbereiche der Jugendforschung in Deutschland öffneten sich auch für visuelle Methoden der Erhebung und Dokumentation. Zu erwähnen sind in diesem Zusammenhang u.a. Foto-Portraits im Rahmen der Shell-Jugendstudie von 1992 (Jugendwerk der Deutschen Shell 1992), einzelne Projekte im Rahmen des DFG-Schwerpunktprogramms „Pädagogische Jugendforschung“ (1980-1986) sowie Projekte der Jugendforschung auf der Basis von Eigenproduktionen mit Video (zusammenfassend: Niesyto 2001a). Diese Eigenproduktionen können als Forschungsdaten genutzt werden; es lassen sich über sie auch weitere verbale Äußerungen anregen. Vor allem dann, wenn die sprachlichen Kompetenzen der Subjekte gering bzw. noch wenig ausgeprägt sind (Kinder, Migranten, Menschen aus benachteiligenden sozialen Milieus), ist es wichtig, non-verbale Äußerungsformen anzubieten. In einer Zeit, in der Wahrnehmung und Welterleben von Kindern und Jugendlichen stark von Medienerfahrungen 
geprägt sind, eröffnet Forschung auf der Grundlage von Eigenproduktionen einen ergänzenden bzw. alternativen Zugang zu deren Lebenswelten.

Der folgende Beitrag fasst im ersten Teil die bisherige Entwicklung des Ansatzes „Eigenproduktionen mit Medien als Gegenstand qualitativer Forschung“ zusammen (Horst Niesyto). Der zweite Teil stellt das aktuelle EU-Forschungsprojekt CHICAM vor - „Children in Communication about Migration“1 (Peter Holzwarth). Das Projekt, das im Herbst 2001 begann, befindet sich derzeit am Beginn der Auswertungsphase. Wir werden anhand der angewendeten Methoden erste Erfahrungswerte vorstellen, die Beispiele für visuelle Methoden im Zusammenspiel mit anderen Methoden der Datenerhebung zeigen.

\section{Qualitative Forschung auf der Basis von Eigenproduktionen mit Medien}

Der spezifische Erkenntnisgewinn visueller Methoden verbindet sich mit der Dokumentation subjektiver Repräsentationsformen, die mit sprachgebundenen Methoden nicht oder nur eingeschränkt möglich sind. So liegt die besondere Qualität des Einsatzes der Fotografie vor allem in der Reduktion auf Momenteindrücke, in der emotional starken Wirkung einzelner Bilder, in der Darstellung räumlicher Kontexte, in projektiven Verfahren (z.B. Fotobefragung). Es gibt auch Ansätze, Fotos nicht nur als Beleg- und Illustrationsmaterial und als Stimuli in biografischen Fotointerviews einzusetzen, sondern Jugendliche und junge Erwachsene zu ermuntern, eigene Fotoaufnahmen zu bestimmten Themen zu machen (vgl. Fuhs 1997, S. 281; 2003, S. 51).

Im Unterschied zur Fotografie ermöglicht die videografische Dokumentation und Gestaltung besonders das Darstellen von Bewegungs- und Handlungsabläufen in Bild und Ton, die Dokumentation komplexer non-verbaler Ausdrucksformen (Mimik, Gestik, Haptik, z.B. in Gruppensituationen), die symbolische Verarbeitung von Erfahrungen und den Ausdruck von Gefühlen und Stimmungen im Zusammenspiel von Bildern, Musik und Sprache. In selbst erstellten Videofilmen haben Jugendliche die Chance, eigene Bilder des Welterlebens über körper- und gegenstandsbezogene sowie über mehr abstrahierende Symbolisierungen auszudrücken. Gerade jenen Jugendlichen, die Probleme mit sprachgebundenen Forschungsmethoden haben, bietet das Medium Video die Möglichkeit, sich mehr auf einer präsentativ-symbolischen Ebene auszudrücken. Besonders emotional besetzte und tabuisierte Themen, Lebensgefühle und Stimmungen können mit eigenem und fremden Bild- und Tonmaterial dargestellt werden (Niesyto 1991, S. 81f; Witzke 2003).

1 Gefördert von der Europäischen Union (5. Rahmenforschungsprogramm). 


\subsection{Mediale Eigenproduktionen - Skizzierung der Forschungssituation}

Die meisten bisherigen Projekte im Bereich der Jugendforschung, die visuelles Material in Forschungsdesigns integrierten, nutzten Fotos vor allem als Belegmaterial und Stimuli im Rahmen von Interviews und Video-Dokumentationen als Alternative zu Tonbandmitschnitten (bei Interview- und Gruppenverfahren). Bei diesen Formen der Datenerhebung werden Visuelles und Audiovisuelles nicht als eigenständige Forschungsstrategie verstanden. Bild- und Filmmaterial dienen lediglich als zusätzlicher Lieferant für Wortund Schriftsprache.

Diese Situation trifft auch für die meisten Studien über die Mediennutzung von Kindern und Jugendlichen zu. So findet sich im „Handbuch Medienpädagogik“ (Hiegemann/Swoboda 1994) nur ein kleiner Abschnitt, der die Notwendigkeit eines „SichÄußern-Könnens“ in visuellen und audiovisuellen Ausdrucksformen im Kontext qualitativer Medien- und Kommunikationsforschung thematisiert (Theunert 1994, S. 399). In einem Band über „Qualitative Kinder- und Jugendmedienforschung“ (Paus-Haase 2000) wird die Notwendigkeit deutlicher betont, stärker non-verbale Artikulationsformen insbesondere in der Kindermedienforschung - einzubeziehen, wobei sich die Beiträge vor allem auf Zeichnen, Malen und Spielaktionen beziehen (Schorb/Theunert 2000, S. 38ff.; Neuß 2000, S. 131ff.).

Das von Yvonne Ehrenspeck und Burkhard Schäffer (2003) herausgegebene Handbuch „Film- und Fotoanalyse in der Erziehungswissenschaft“ versammelt zwar eine Vielzahl von Beiträgen zur Theorie, Methodik und praktischen Anwendungsbereichen der Foto- und Filmanalyse und arbeitet die spezifischen Qualitäten visueller und audiovisueller Materialien als Quelle wissenschaftlicher Erkenntnis heraus. Aber auch diese wichtige, neue Veröffentlichung erwähnt nur am Rande die Möglichkeiten und Vorteile von medialen Eigenproduktionen von Kindern und Jugendlichen (vgl. Fuhs 2003, S. 51f.).

Erstaunlich ist auch die Situation im Bereich der „Cultural Studies“, zumindest im deutschsprachigen Raum. Die Studien beziehen sich nahezu ausschließlich auf die Erforschung von Rezeptionsprozessen bei massenmedialen Produkten (vgl. Hepp 1999, Hepp/Winter 1999). Ausnahmen sind z.B. Aspekte der Studie von Vogelgesang (1991) über die kulturell-medialen Praktiken von Video-Cliquen.

Insgesamt mangelt es in der pädagogischen und in der medienpädagogischen Forschung an einer systematischen Reflexion über die Möglichkeiten, Eigenproduktionen von Kindern und Jugendlichen in Forschungsdesigns zu integrieren und als spezifische Quelle für wissenschaftliche Forschung zu nutzen. Forschungsmethoden in der Medienpädagogik berücksichtigen bislang sehr zurückhaltend visuelle Methoden der Datenerhebung und der Datenauswertung. Dies ist umso erstaunlicher, als Vertreter der Medienpädagogik seit vielen Jahren einen „Wahrnehmungswandel“ bei Kindern und Jugendlichen konstatieren (Baacke 1997) und von einer „Medienkindheit“ und „Medienjugend“ sprechen (Charlton/Neumann 1992). Gleichzeitig ist zu beobachten, dass es zwar eine 
Reihe von Medienrezeptionsstudien gibt, allerdings nur relativ wenige Studien, die systematisch die Praxis der Medienarbeit untersuchen.

Die Brisanz, Eigenproduktionen mit Medien stärker einzubeziehen, ergibt sich vor allem aufgrund von Veränderungen in der Art und Weise der Wahrnehmung und Aneignung von Wirklichkeit bei Kindern und Jugendlichen. Folgende Aspekte sind hervorzuheben:

- Die sich mit der modernen Medienentwicklung herausbildenden neuen Formen raumzeitlicher Weltwahrnehmung sowie der verstärkten Vermischung von realen und fiktiven Situationen, von face-to-face und medienvermittelter Kommunikation.

- Die besonders mit audiovisuellen Medienangeboten verbundenen affektiven und emotionalen Erlebnisformen (insbesondere im Bereich präsentativer Symbolik).

- Die Veralltäglichung des Medialen und die wachsende Bedeutung der Medienkommunikation für die Identitätsentwicklung und die Lebensbewältigung insgesamt.

Wenn es stimmt, dass die Medienförmigkeit von Wahrnehmungsprozessen an Bedeutung gewonnen hat, kann dies nicht ohne Auswirkung auf theoretischkonzeptionelle Überlegungen bei der Entwicklung von Forschungsdesigns bleiben. Da Rezeptionsformen - so die Annahme - auch Ausdrucksformen beeinflussen, geht es zugleich um methodische Fragen, gerade bei subjektorientierten Forschungsansätzen. Diese Ansätze legen großen Wert auf sog. subjektadäquate Methoden der Repräsentation. Die These ist: Wer in der heutigen „Mediengesellschaft“ etwas über die Vorstellungen, die Lebensgefühle, das Welterleben von Kindern und Jugendlichen erfahren möchte, sollte ihnen die Chance bieten, sich - ergänzend zu wort- und schriftsprachlichen Formen - auch mittels eigener, selbst produzierter Medien und damit verbundener präsentativ-symbolischer Formen ${ }^{2}$ auszudrücken.

Forschung hat die Möglichkeit, die Produktionsprozesse (Symbolproduktion) zu dokumentieren und verschiedene Ausdrucksformen, präsentative (Bilder, Musik, Körpersprache) und diskursive (Wort- und Schriftsprache) zu analysieren und zu interpretieren. Dabei ist zu differenzieren zwischen Eigenproduktionen, die ohne und die mit Beratung von Medienpädagogen und -pädagoginnen bzw. anderen Berater und Beraterinnen entstehen. Eigenproduktionen im Kontext medienpädagogischer Beratung sind ein wichtiger Teil medienpädagogischer Forschung.

Theoretisch-konzeptionell beziehen sich die eigenen Studien vor allem auf

- jugendkulturtheoretische Überlegungen, die milieuspezifische Aspekte nicht außer Acht lassen,

- symboltheoretische Überlegungen, die an den Arbeiten von Ernst Cassirer, Susanne Langer und sozialpsychologischen Symboltheorien anknüpfen,

2 Der Begriff „präsentativ-symbolisch“ geht auf Arbeiten von Susanne Langer (1942) zurück; vgl. Belgrad/ Niesyto (2001b, S. 7f). 
- medienpädagogische Theorien, die sozial-ästhetische Dimensionen akzentuieren (vgl. Niesyto 2001c, S. 55ff.).

Inzwischen gibt es in Deutschland und im Ausland mehrere Studien, insbesondere über Eigenproduktionen mit Video (zusammenfassend: Niesyto 2000a, S. 137ff.; 2001b, S. 92ff.). Die spezielle Forschungsperspektive „Eigenproduktionen mit Video als Gegenstand der Jugendforschung“ ist mit methodologischen und methodischen Fragen und Herausforderungen verbunden, die sich vor allem auf die Einschätzung der spezifischen Qualität dieser „medialen Selbstzeugnisse“ (u.a. Ausdrucksmöglichkeiten der Produzenten und Produzentinnen, Einflüsse durch Medienpädagogen und -pädagoginnen) sowie ihrer Deskription, Analyse und Interpretation beziehen (u.a. Umgang mit Mehrdeutigkeiten, Verstehen latenter Symbolisierungen, Transferproblematik Bild/Schriftsprache; vgl. Niesyto 1991, S. 212f.; 1999, S. 336ff.; 2000a S. 145ff.). Auch lassen sich unterschiedliche ästhetische Formen von Eigenproduktionen unterscheiden, die sich aus den spezifischen Fragestellungen, Gegenstandsbereichen und Ausdruckspotentialen der Kinder/Jugendlichen ergeben (Niesyto 2000a, S. 150f.; 2001b, S. 97ff.).

Entscheidend ist in jedem Fall, dass die Eigenproduktionen von Kindern und Jugendlichen selbst gemacht werden, dass Mädchen und Jungen mittels Medien ihre Themen, Gefühle, Phantasien, Erfahrungen ausdrücken können. Eigenproduktionen sind situations- und prozessbezogene Momentaufnahmen aus der Alltags- und Lebenswelt von Kindern und Jugendlichen, die unterschiedliche Ausdrucksintensitäten und Grade von Reflexivität enthalten. Diese sind vor allem abhängig von Alter, vorhandenen medialen Kompetenzen, unterstützenden Anregungsmilieus sowie der Qualität von Forschungsdesigns, insbesondere der Offenheit im methodischen Vorgehen. Diese Offenheit ist ein Spezifikum qualitativer Forschungsansätze (vgl. Mayring/Gläser-Zikuda/Hurst 2001, S. 17f.). Es geht darum, Kindern und Jugendlichen Formen des Selbstausdrucks zu ermöglichen, die an ihren vorhandenen Ausdruckskompetenzen ansetzen und ihnen kommunikative Spielräume eröffnen.

Der methodologischen Diskussion im Bereich der Jugendtagebuchforschung ist zu entnehmen, dass die in Tagebüchern festgehaltenen Eindrücke, Erlebnisse, Reflexionen kulturell eingebettete Selbstzeugnisse darstellen, die nicht ohne hermeneutische Deutungsarbeit wissenschaftlich verwendet werden können (Winterhager-Schmid 1997). Die Erschließung der Interdependenzen von Individuierung und Enkulturation ist eine Aufgabe, die sich auch und gerade bei Selbstzeugnissen stellt: „Gerade weil Jugendtagebücher Zeugnisse virtueller Reflexivität und noch changierender Selbstentwürfe sind, können sie eher aufgefasst werden als das, was Ricoeur als „meaningful action“ charakterisiert hat; d.h. sie sind Schreibhandlungen mit Bedeutung für das schreibende Subjekt“ (Winterhager-Schmid 1997, S. 364). Es geht um vorläufige Selbstbilder, um ausschnitthafte Einblicke in Identitätsbildungsprozesse, die auf dem Hintergrund altersspezifischer Bewältigungsaufgaben und soziokultureller Kontexte zu verstehen sind. 
Die Methode der Tagebuchforschung wird inzwischen in verschiedenen Bereichen angewendet und mit anderen Methoden verknüpft, insbesondere mit verschiedenen Beobachtungs- und Befragungsformen. Es gibt auch Überlegungen, Spracharbeit zu verfeinern und die symbolische Ordnung der Sprache an ihren Gegenstand anzupassen. Hierzu gehört ein Sich-Einlassen auf Texte, die in Form von Gedichten oder Erlebnisaufsätzen entstehen. Die Selbstäußerungen können dabei auch poetisch-metaphorische Sprachbilder enthalten, die emotionale und imaginative Erlebnisqualitäten Jugendlicher auf unterschiedlichen Abstraktionsniveaus ausdrücken, z.B. im Rahmen „freien Schreibens“ ohne Vorgabe einer bestimmten Textsorte (vgl. Fix/Melenk 2000).

Die Orientierung auf schriftsprachliche und verbale Ausdrucksformen enthält jedoch Begrenzungen. So stellt sich in der Kindheits- und Kindermedienforschung vor allem aus altersspezifischen Gründen die Notwendigkeit, non-verbale Methoden wie Rollenspiele, Malen, Gestalten einzubeziehen, da Kindern Wort und Schrift nur begrenzt zur Verfügung stehen (vgl. Neuß 1999; Paus-Haase 2000, S. 24ff.; Schorb/Theunert 2000, S. 42ff.). Des Weiteren ist bekannt, dass Tagebücher vor allem ein Ausdrucksmedium von Mädchen und jungen Frauen sind und weniger von Jungen genutzt werden (Winterhager-Schmid 1997, S. 361). Aus der pädagogischen bzw. medienpädagogischen Praxisforschung gibt es schließlich diverse Hinweise, dass eine Reduktion auf Formen des schriftlichen und verbalen Selbstausdrucks für Kinder und Jugendliche aus sozialund bildungsmäßig benachteiligenden Verhältnissen oft mit Schwierigkeiten verbunden sind (Niesyto 1991, S. 155ff; 2000b, S. 12f.).

\subsection{Unterschiedliche Formen von Eigenproduktionen in Forschungskontexten}

Differenzierend sind folgende Formen einer qualitativen Forschung auf der Basis von Eigenproduktionen im Bereich Video zu unterschieden:

a) Kinder/Jugendliche nutzen das Medium Video für eigene kulturelle und kommunikative Praxisaktivitäten, die ohne Unterstützung durch Medienpädagogen und -pädagoginnen oder andere Berater/Beraterinnen zustande kommen. Forscher und Forscherinnen analysieren und interpretieren die Filme. Hier gibt es in der Forschungsarbeit wiederum unterschiedliche Formen:

- Forscher/Forscherinnen haben die Möglichkeit, beim Entstehen und beim kommunikativen Austausch der Produktionen dabei zu sein und können Kontexte dokumentieren;

- Forscher/Forscherinnen haben nur einen Zugang zu den Filmen und rekonstruieren einzelne Kontextdaten nachträglich.

b) Kinder/Jugendliche nutzen das Medium Video für Eigenproduktionen, die sie mit Unterstützung von Medienpädagogen/-pädagoginnen oder anderen Berater/Beraterinnen erstellen. In diesem Fall ist es besonders wichtig, dass 
die Forschung die Entstehungsbedingungen, insbesondere die Interaktionen zwischen Jugendlichen und Berater/Beraterinnen, dokumentiert und reflektiert.

c) Forscher/Forscherinnen erstellen zusammen mit Kindern/Jugendlichen und Filmemachern/ -macherinnen Videofilme in einem dialogischen Verfahren. Kinder/Jugendliche sind in Teilbereichen, z.B. bei den Aufnahmen, aktiv beteiligt und artikulieren ihre Vorstellungen zur Auswahl und zur Montage der Aufnahmen.

Diese verschiedenen Formen sind als Möglichkeitstypen zu verstehen. In der Forschungspraxis gibt es Mischformen, die in unterschiedlicher Intensität z.B. ethnografisch-explorative mit medienpädagogischen Intentionen verbinden. Nach unserem Verständnis gehören Forschungsprojekte des Typs a) zum Kernbereich ethnografischer Forschung: Der Fokus liegt auf der Dokumentation, Analyse und Interpretation von Aneignungsprozessen, ästhetischen Ausdrucksformen, Symbolisierungen, kommunikativen Prozessen, die bei einzelnen Kindern/Jugendlichen oder Gruppen beim Umgang mit dem Medium Video beobachtet werden können. Hierfür sind explorative Forschungsmethoden geeignet, die sich in der Feldforschung bewährt haben: Teilnehmende Beobachtung, Gruppengespräche/-diskussionen, Videofilm- und Dokumentenanalysen und eventuell zusätzliche Leitfaden-Interviews. Offenheit und Flexibilität im Methodeneinsatz sind besonders wichtig, um die Eigendynamiken beim Produktionsprozess nicht zu stören.

Forschungsprojekte des Typs b) können ethnografische Elemente enthalten, sind jedoch im Kern Projekte medienpädagogischer Forschung. Zu dokumentieren und zu reflektieren sind Formen der ästhetischen, inhaltlichen, technischen und gruppenpädagogischen Einflussnahme, die auch bei zurückhaltenden, non-direktiven Beratungsformen erfolgt. Im Unterschied zu medienpädagogischen Evaluationsprojekten, bei denen konkrete medienpädagogische Konzepte, Ziele und Arbeitsformen systematisch überprüft werden, möchte der Ansatz „medienpädagogische Forschung“ im Rahmen bestimmter medienpädagogischer Arrangements einen Zugang zu Lebens- und Medienwelten Jugendlicher erhalten und ist zugleich an der Reflexion und Weiterentwicklung medienpädagogischer Konzepte interessiert. Bei diesem Forschungstyp (vgl. Niesyto 1991, 1996, 2003) ist die systematische Erhebung und Reflexion von Kontextinformationen während des Produktionsprozesses unerlässlich, um die Interaktionen zwischen Jugendlichen und Pädagogen/Pädagoginnen (und selbstverständlich auch Forscher/Forscherinnen) so genau wie möglich zu dokumentieren. Das Agieren von Pädagogen/Pädagoginnen im Kontext medienpädagogischer Forschung bedarf sorgfältiger Vorbereitung und Begleitung, um durch einen einfühlsamen Stil der Beratung subjektive Formen des symbolischen Ausdrucks zu gewährleisten. Medienpädagogische Forschung erfüllt nur dann ihren Zweck, wenn Kinder/Jugendliche durch Video Ausdrucksmöglichkeiten erhalten, um ihre Themen, Erfahrungen, Phantasien auszudrücken. Subjektorientierung, Prozessorientierung, methodische Offenheit und Flexibilität sind wichtige 
Grundsätze, um die Medienpraxis von Kindern/Jugendlichen nicht „heimlichen Lehrplänen“ zu subsumieren. Es ist zwar notwendig, dass die Forschung entsprechend der Forschungsfragestellung und der Definition des Gegenstandsbereichs einen gewissen Rahmen vorgibt. Die Ausgestaltung dieses Rahmens liegt jedoch bei den Kindern/Jugendlichen und darf nicht durch pädagogische „Zwänge“ eingeengt und strukturiert werden.

\subsection{Methodologisch-methodische Erfahrungswerte}

Auf dem Hintergrund der Bilanzierung mehrerer Forschungsprojekte (zusammenfassend: Niesyto 2001a) sind unter methodologisch-methodischer Perspektive folgende Punkte hervorzuheben:

\section{Dimension des Erstellens von Eigenproduktionen}

- Eigenproduktionen mit Medien sollten Kindern und Jugendlichen Chancen eröffnen, um die spezifische Qualität präsentativer Symbolisierungen nutzen zu können. Hierfür bedarf es der Weiterentwicklung kreativer Konzepte zur Förderung präsentativsymbolischen Selbstausdrucks mit Medien, die sich am Grundsatz subjektiver Stilund Symbolbildung orientieren (vgl. Maurer 2001a, b).

- Der Forschungsansatz bezieht sich auf das Erstellen von Medienproduktionen in Gruppenkontexten. Dies setzt voraus, möglichst alle Gruppenmitglieder in den Produktionsprozess einzubeziehen und die Formen des arbeitsteiligen Erstellens und die damit verbundenen Kommunikations- und Aushandlungsprozesse genau zu beobachten und zu dokumentieren (Einfluss individueller Beiträge auf das gemeinschaftlich produzierte Produkt).

- Die bisherigen Projekte verwendeten vor allem das Medium Video. Sinnvoll erscheint eine Ausdifferenzierung der Möglichkeiten, welche Medien und welche medialen Ausdrucksformen (Genres, multimediale Arrangements) im Kontext welcher Fragestellung am sinnvollsten sind. Hierzu gehört auch die Frage nach dem Zusammenspiel von wort- und schriftsprachlichen Ausdrucksformen mit Bildern, Musik und körpersprachlichen Ausdrucksformen (vgl. Niesyto 2001b, S. 97ff.).

- Die vorhandenen Forschungsressourcen haben Auswirkungen auf Umfang und Intensität der zu begleitenden und auszuwertenden Eigenproduktionen. Bei geringeren Forschungsmitteln ist darauf zu achten, einfachere Formen von Eigenproduktionen zu wählen, deren Erstellung weniger zeit- und beratungsintensiv ist. In jedem Fall ist zu gewährleisten, dass medienpädagogische Beratung und wissenschaftliche Begleitung nicht von derselben Person geleistet werden.

- Bei Eigenproduktionen, die in pädagogisch arrangierten Feldern entstehen, sind die Interaktionsprozesse zwischen Erwachsenen (Forscher/Forscherinnen, Pädagogen/ Pädagoginnen, anderen professionellen Berater/Beraterin- 
nen) und Kindern bzw. Jugendlichen genau zu dokumentieren und bei der Datenauswertung zu analysieren.

- Künftige Forschungsprojekte sollten stärker Eigenproduktionen beachten, die außerhalb von pädagogischen Arrangements entstanden sind. Dies vereinfacht nicht unbedingt den Zugang zu Kontextdaten, ist aber wichtig, um den Grundsatz der Alltagsorientierung einzulösen sowie Übertragungsprozesse aufgrund pädagogischer Interaktionen zu minimieren (vgl. Schmidt 2001).

- Die einzelnen Forschungsschritte sind genau zu dokumentieren, ausgehend von der klaren Formulierung der Fragestellung und der dahinter stehenden Theorie, über die genaue Beobachtung der Produktionsprozesse bis hin zur Offenlegung der einzelnen Auswertungsschritte sowie der Rückbeziehung der gewonnenen Ergebnisse auf die Theorie.

Dimension der Analyse und der Interpretation von Eigenproduktionen

- Verstehensprozesse lassen sich nur bis zu einem gewissen Grade objektivieren. Umso wichtiger ist es, die jeweiligen Bezugstheorien offen zu legen, um insbesondere Stellenwert und Reichweite von Interpretationen besser einschätzen zu können.

- Präsentative Symbolisierungen erfordern andere Formen der Analyse und Interpretationen als wort- und schriftsprachliches Material. Methoden sind zu fördern, die sich assoziativ dem präsentativen Material annähern (z.B. Ersteindrucksanalysen) und eine Vielzahl von Lesarten sammeln, um sie vergleichend auszuwerten (kommunikative Validierung $)^{3}$ und mit Kontextinformationen abzugleichen.

- Notwendig ist ein systematisches Vorgehen bei der Analyse der Eigenproduktionen. Hierfür reichen „klassische“ Kriterien der Bild-, Film- und Musikanalyse nicht aus. Sinnvoll erscheinen Kombinationen, die sich zwar auf klassische Analyseinstrumentarien beziehen, zugleich aber kinder- und jugendkulturelle Aspekte beim Umgang mit Hör- und Bildmedien berücksichtigen. Hier gibt es einen besonderen Bedarf zur Weiterentwicklung entsprechender Methoden und Analyseverfahren.

- Latente Sinnschichten sind stets im Zusammenhang mit manifesten Botschaften herauszuarbeiten, um Gefahren einer Überinterpretation entgegenzuwirken. Diskursiv und präsentativ orientierte Methoden sind zusammen einzusetzen, um einen Prozess der reflexiven Bedeutungserschließung zu gewährleisten.

- Ergebnisse aus Forschungsprojekten, die sich auf Eigenproduktionen mit Medien beziehen, sollten im Sinne intersubjektiver Überprüfbarkeit auch den Gegenstand der Forschung - die Eigenproduktionen - Dritten zugäng-

3 Unter „kommunikativer Validierung“ verstehen wir in diesem Zusammenhang weniger Formen der retrospektiven Interpretation der Medienproduktionen durch die jeweiligen Produzenten/Produzentinnen (Kinder, Jugendliche), sondern primär das Einbeziehen mehrerer Forscher/Forscherinnen in den Analyse- und Interpretationsprozess. 
lich machen. Dies bedeutet, nicht nur in Schriftform Projekte zu dokumentieren, sondern zugleich präsentativ-symbolische Materialien zu veröffentlichen (vgl. die CDROM Dokumentation über das Projekt „VideoCulture“, Niesyto 2003).

- Künftige Projekte sollten den praktischen Verwendungszweck von Forschung noch stärker beachten, um konkrete, lebensweltbezogene Situationen und Probleme von Kindern und Jugendlichen besser verstehen und Handlungsalternativen entwickeln zu können.

\section{Das EU-Forschungsprojekt CHICAM - Children in Communication about Migration}

Das Projekt CHICAM knüpft im methodischen Bereich an Erfahrungen des internationalen Projekts VideoCulture (Niesyto 2003) an und verbindet eine ethnografisch-lebensweltliche Dimension (Exploration von Lebenswelten junger Migranten/Migrantinnen) mit einer medienpädagogischen Dimension (Förderung von Medienkompetenzen).

\subsection{Gegenstand - Konzept - Ziele}

Das Projekt CHICAM möchte 10- bis 14-jährigen Kindern, die im Kontext von Migration oder Flucht in verschiedenen Ländern Europas leben, die Möglichkeit geben, sich mittels Fotografie, Video und Internet mit der eigenen Lebenssituation auseinander zu setzen (Peergroup-Beziehungen, Familienbeziehungen, Schule) und Erfahrungen über Ländergrenzen hinweg auszutauschen (vgl. Holzwarth/Maurer/Niesyto 2002). ${ }^{4}$

Das Projekt verfolgt das Ziel, das Potential neuer Medien für interkulturelle Kommunikation, Reflexion und Integration zu untersuchen. Mit der Vermittlung von Medienkompetenz und Ausdrucks- und Reflexionsfähigkeit sollen die Kinder zudem zu gesellschaftlicher Teilhabe befähigt werden. Es ist geplant, die Produktionen der Kinder unterschiedlichen Öffentlichkeiten zugänglich zu machen, um im sozialen Umfeld der Kinder, in lokalen Institutionen z.B. Schulen) und in politischen Institutionen ein stärkeres Bewusstsein für die Lage von Kindern aus Migrations- und Fluchtkontexten zu schaffen.

In den beteiligten Ländern (Großbritannien/London, Italien/Rom, Niederlande/ Utrecht, Schweden/Stockholm, Griechenland/Athen und Deutschland/Ludwigsburg) wurden Gruppen von Kindern, die sog. „CHICAM-Clubs“ gebildet. Diese ethnisch und geschlechtlich gemischten Gruppen (ca. acht bis zwölf Kinder mit relativ aktueller Migrationserfahrung) arbeiteten mit digitaler

4 Das Projekt wurde von Prof. Dr. David Buckingham und Dr. Liesbeth de Block von der University of London, Institute of Education, konzipiert und koordiniert. 
und analoger Fotografie sowie mit Video und kommunizierten mit ihren Partnergruppen über eine speziell eingerichtete Internetplattform. Die Gruppen wurden jeweils von einem Medienpädagogen bzw. einer Medienpädagogin begleitet. Eine weitere Person arbeitete in den jeweiligen Clubs, um die Produktions- und Kommunikationsprozesse wissenschaftlich zu begleiten und zu dokumentieren und um die Lebenswelten der Kinder zu erforschen. ${ }^{5}$ Zusätzlich zu Diskussionen und Besprechungen in der internationalen und lokalen Projektgruppe wurden die praktische Medienarbeit und die Forschungsarbeit im Rahmen von Supervision reflektiert.

Der CHICAM Club in Deutschland ist in das Ganztagesbetreuungsangebot einer Hauptschule integriert. Diese Hauptschule befindet sich im Zentrum einer mittelgroßen Stadt in Süddeutschland und weist einen hohen Anteil an Schülerinnen und Schülern mit Migrationshintergrund auf ( $80 \%$ mit Russlanddeutschen und Vorbereitungsklassen; 27 verschiedene Länder). Von 270 Schülern haben 180 eine andere Staatsbürgerschaft als die deutsche. Im CHICAM-Club trafen sich von Oktober 2002 bis Juli 2003 einmal pro Woche von 14 bis 16 Uhr acht Kinder aus der Türkei, Kuba, der Dominikanischen Republik und Tunesien. Zusätzlich zu den Clubtreffen fanden mehrere Intensivphasen, Ausflüge und Intranettreffen statt.

Die Clubmitglieder bestanden aus fünf Mädchen zwischen 13 und 15 Jahren und drei Jungen zwischen 12 und 14 Jahren.

Die Clubmitglieder besuchten alle zusammen eine so genannte Vorbereitungsklasse, eine speziell eingerichtete Schulklasse für Kinder, die noch nicht über ausreichende Sprachkompetenzen verfügen, um eine Regelklasse zu besuchen. Die Projektarbeit begann mit der Sichtung und Aufarbeitung von bisherigen Forschungs- und Praxisprojekten im Bereich Kinder, Migration und Medien. In jedem der beteiligten Länder wurde je ein Bericht zu den Themen „Children and Migration“ sowie „Children and Media“ produziert (für die Bundesrepublik Deutschland: Holzwarth 2002 und Holzwarth/Maurer 2002). Länderübergreifend entstanden zu den beiden Themenbereichen Zusammenfassungen (Buckingham 2002; Westin 2002).

Die Forschungsbereiche visuelle Kommunikation, Peergroup-Beziehungen, Familienbeziehungen sowie Schule, Bildung und Arbeit sind gemäß den länderspezifischen Vorerfahrungen und Kompetenzen aufgeteilt. In allen Ländern wurden Daten zu den genannten Themenfeldern erhoben (Medienproduktionen, Interviews und Feldbeobachtungen), wobei jeweils zwei Projektpartner schwerpunktmäßig für einen Bereich zuständig sind. Auf der Basis von Fallstudien werden thematische Analysen und summative Analysen erstellt. Die Medien-

5 Björn Maurer (Diplompädagoge und Lehrbeauftragter an der PH Ludwigsburg) übernahm die medienpädagogische Begleitung des Projekts. Peter Holzwarth (Diplompädagoge und wissenschaftlicher Angestellter an der PH Ludwigsburg) war für die wissenschaftliche Begleitung der Projektpraxis vor Ort zuständig. Die Club-Aktivitäten und die gesamte Projektentwicklung wurden in regelmäßigen Abständen mit Prof. Dr. Horst Niesyto (lokale Projektleitung) reflektiert. 


\begin{tabular}{|l|l|l|l|l|}
\hline Name $^{6}$ & Alter & Herkunftsland & Muttersprache & $\begin{array}{l}\text { Aufenthalt in } \\
\text { Deutschland }\end{array}$ \\
\hline Serife (w) & 15 & Türkei & Türkisch/kurdisch & ca. 1 Jahr \\
\hline Meral (w) & 15 & Türkei & Türkisch/kurdisch & ca. 1 Jahr, 6 Monate \\
\hline Taskania (w) & 14 & Dominikanische Republik & Spanisch & ca. 1 Jahr, 2 Monate \\
\hline Fatma (w) & 14 & Türkei & Türkisch & ca. 1 Jahr \\
\hline Susan (w) & 13 & $\begin{array}{l}\text { Geboren in Deutschland, } \\
\text { mit 2 Jahren in die USA, } \\
\text { mit 12 Jahren zurück nach } \\
\text { Deutschland }\end{array}$ & Englisch & ca. 7 Monate \\
\hline Mustafa (m) & 14 & $\begin{array}{l}\text { Geboren in Deutschland, } \\
\text { mit 4 Jahren nach Tunesien, } \\
\text { mit 14 Jahren zurück nach } \\
\text { Deutschland }\end{array}$ & Tunesisch & ca. 9 Monate \\
\hline Hakan (m) & 13 & Türkei & Türkisch & ca. 1 Jahr \\
\hline Fernando (m) & 12 & Kuba & Spanisch & ca.1 Jahr, 10 Monate \\
\hline
\end{tabular}

Produktionen der Kinder stellen nicht nur wichtige Daten für die Forschung dar, sie sollen auch dazu dienen, Themen und Aussagen der Kinder in verschiedenen sozialen und politischen Öffentlichkeiten zugänglich zu machen.

Einige Partner verfügen über spezielle Erfahrungen im Bereich Migrationsarbeit und -Forschung, andere dagegen stärker im Bereich Medienpädagogik und Medienforschung. Dadurch ergibt sich eine interessante Kooperationssituation, in der die unterschiedlichen Partner von der jeweiligen Expertise der anderen profitieren können. Interkulturelles und interdisziplinäres Lernen findet also auch auf der Ebene der Forschungsund Praxisarbeit statt.

Das „Institute of Education“ an der „University of London“ ist zusammen mit der Pädagogischen Hochschule Ludwigsburg/Abteilung Medienpädagogik für den Bereich visuelle Kommunikation zuständig. Beide Partner verfügen über Vorerfahrungen aus dem internationalen Projekt VideoCulture, das von 1997 bis 2001 durchgeführt wurde. Im Rahmen des Projekts hatte sich gezeigt, dass sich Jugendliche aus verschiedenen europäischen Ländern und den USA aufgrund partiell geteilter Medienkulturen über Sprachgrenzen hinweg mit Bildern und Musik verständigen konnten. Bei den beteiligten Jugendlichen spielten im Rahmen der Interpretation und Aneignung von Videos nicht nur inhalts- und themenorientierte Zugänge eine Rolle. Wichtig waren auch emotionale, ästhetische und assoziative Aneignungsweisen, bei denen Gefühle des Angezogen-Seins wichtig waren sowie die Verbindungen zwischen eigenen Erfahrungen und Aspekten des Films (Holzwarth/Maurer 2003; Niesyto 2003).

Im Rahmen von CHICAM sollen die Beobachtungen aus VideoCulture anhand einer jüngeren Altersgruppe, die den speziellen Erfahrungshintergrund Migration teilt, erweitert werden. Folgende Fragestellungen sind im Rahmen

6 Die Namen der CHICAM-Clubmitglieder sowie weiterer am Projekt beteiligter Personen (Lehrerin und Lehrer) sind geändert. 
des Forschungsschwerpunkts „visuelle Kommunikation“ vor allem von Interesse:

1) Welche Medienerfahrungen haben die beteiligten Kinder und Jugendlichen?

2) Aus welchen kulturellen Symbolkontexten bedienen sich Kinder im Rahmen der Kommunikationsprozesse (Herkunftsland, Einwanderungsland, globale Kulturen)? Inwiefern werden Formen visueller Sprache entwickelt?

3) In wieweit lassen sich im Rahmen der medienpädagogischen Arbeit mit Hilfe eines speziellen Projekt-Intranets Formen interkultureller Kommunikation ermöglichen?

4) Welche medienpädagogischen Konzepte und Angebote eignen sich für die Zielgruppe Kinder/Jugendliche mit Migrationshintergrund und fördern interkulturelle Kommunikation?

Das „Centre for Research in International Migration and Ethnic Relations (CEIFO)“ in Stockholm und das „Greek Council for Refugees“ in Athen sind federführend für den Arbeitsbereich Peergroup-Beziehungen verantwortlich. Es geht dabei um die Beziehungen innerhalb der Produktionsgruppen, die interkulturellen medienvermittelten Beziehungen zwischen den Ländergruppen und den Beziehungen, die die Gruppenmitglieder zu anderen sozialen Kontexten oder Institutionen haben.

Die folgende Darstellung zeigt die thematischen Bereiche des Projekts sowie die schwerpunktmäßigen Zuständigkeiten der Projektpartner.

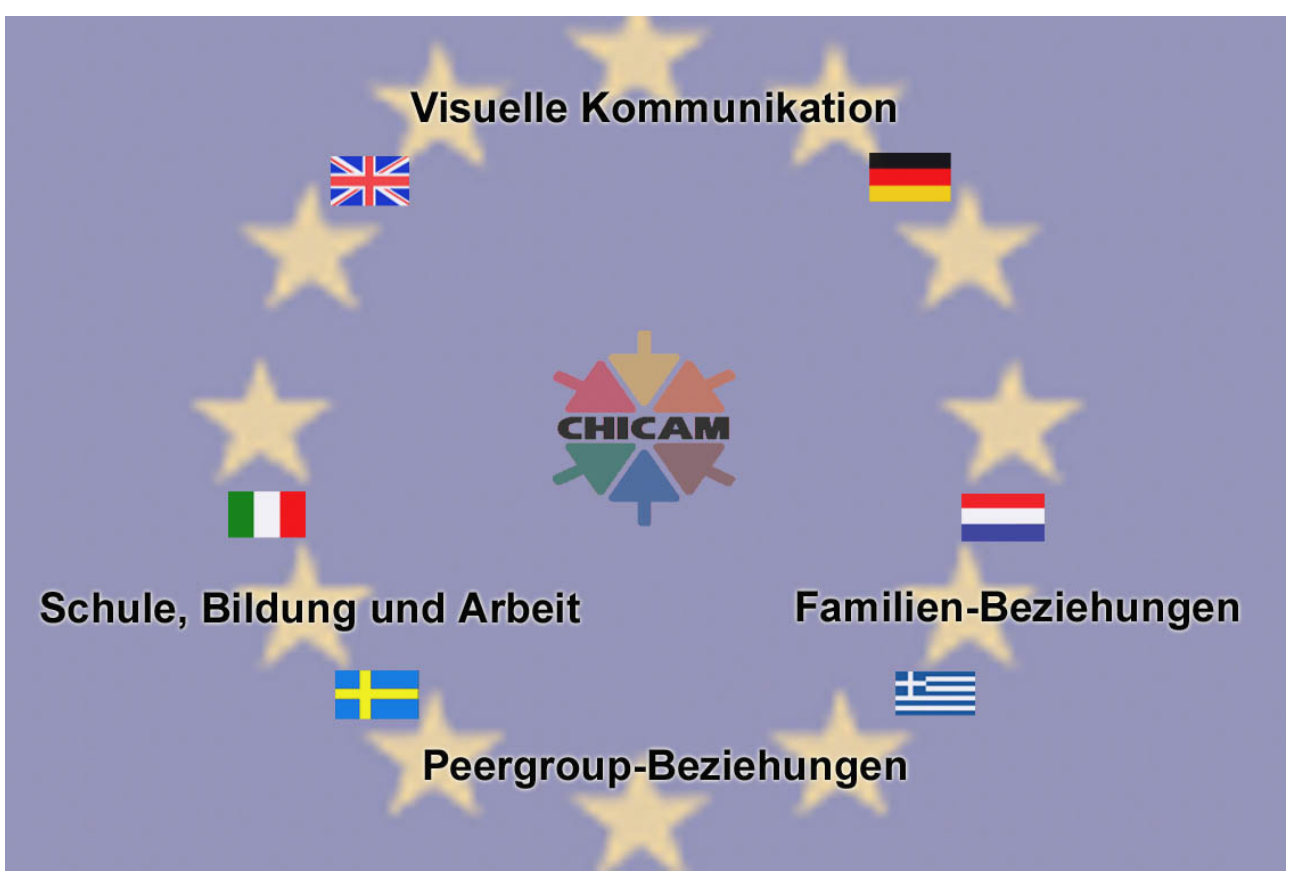

Abb. 1: Thematische Bereiche und Zuständigkeiten der CHICAM-Partnerländer. 
Das Forschungsfeld Familienbeziehungen wird schwerpunktmäßig von den Partnern „Greek Council for Refugees“ in Athen und „FORUM Institute of Multicultural Development“ in Utrecht bearbeitet. Im Kontext von Kindern mit Migrationshintergrund wird häufig eine Problem- und Defizitperspektive eingenommen, ihre Existenz wird oft als ein problematisches „Zwischen-den-Kulturen-Leben“ aufgefasst. Es ist jedoch wichtig auch die Kompetenzen wahrzunehmen, die im Schnittfeld verschiedener kultureller Bezugskontexte entstehen können.

Für den Bereich Schule, Bildung und Arbeit sind in erster Linie die Partner „Centre for Research in International Migration and Ethnie Relations (CEIFO)“ in Stockholm und „Fondazione Centro Studi Investimenti Sociali (CENSIS)“ in Rom zuständig. Schule kann auf der einen Seite Integration und gesellschaftliche Teilhabe ermöglichen, auf der anderen Seite besteht die Gefahr, dass sie Kompetenzen von Minderheiten ignoriert und Ausgrenzungsprozesse verstärkt. Der Lernort Schule stellt eine große Chance für interkulturelle Kommunikation, interkulturelles Lernen und Integration dar. Spracherwerb und Sprachvermittlung sind zentrale Herausforderungen für die Schule.

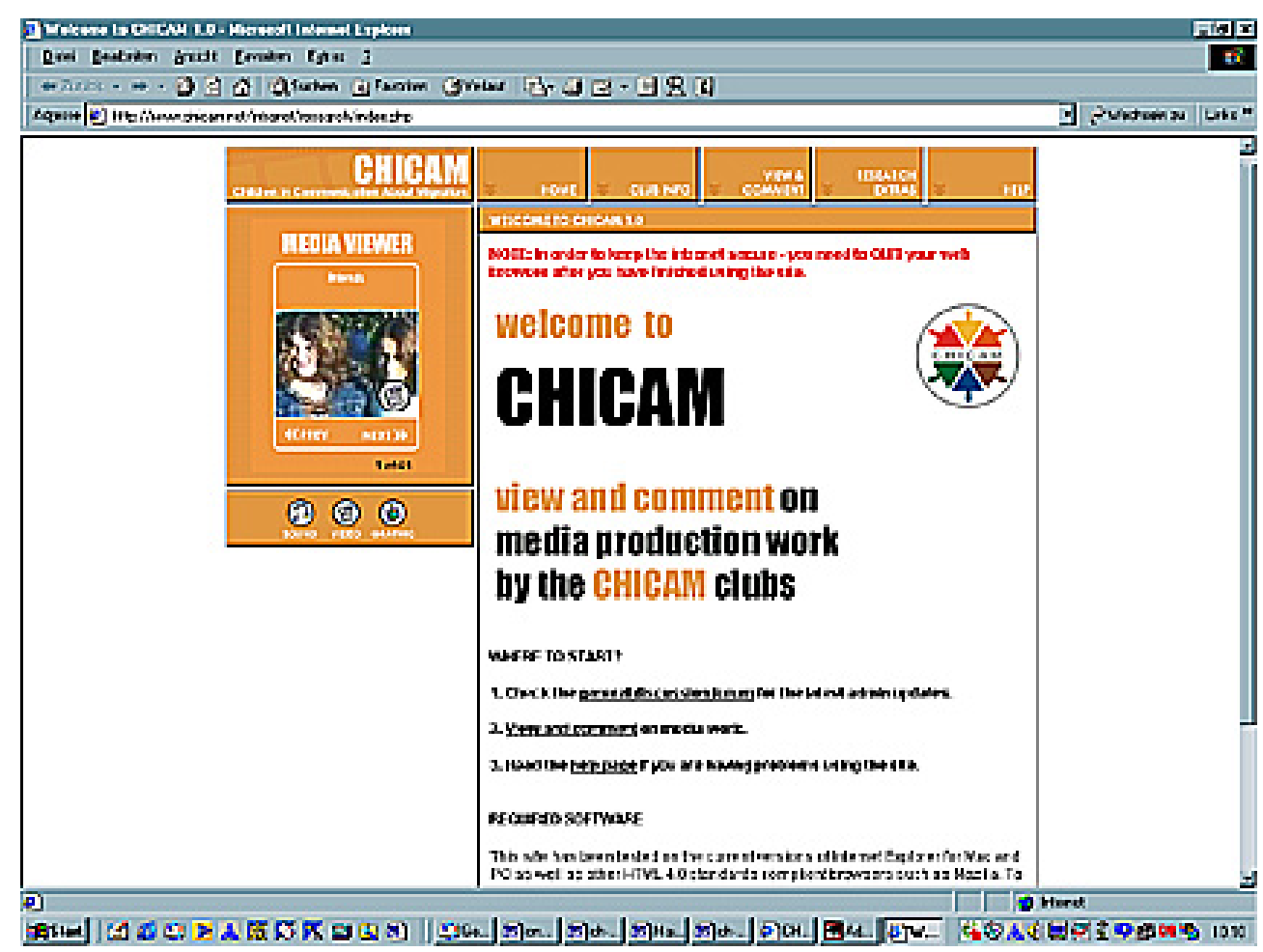

Abb. 2: Screenshot der CHICAM Researcher-Intranetplattform. 
Kommunikation zwischen den Projektpartnern findet face-to-face auf Forschungstreffen und bilateralen Treffen statt, sowie medienvermittelt über eine speziell eingerichtete CHICAM Researcher-Intranetplattform (vgl. Abbildung 2) ${ }^{7}$.

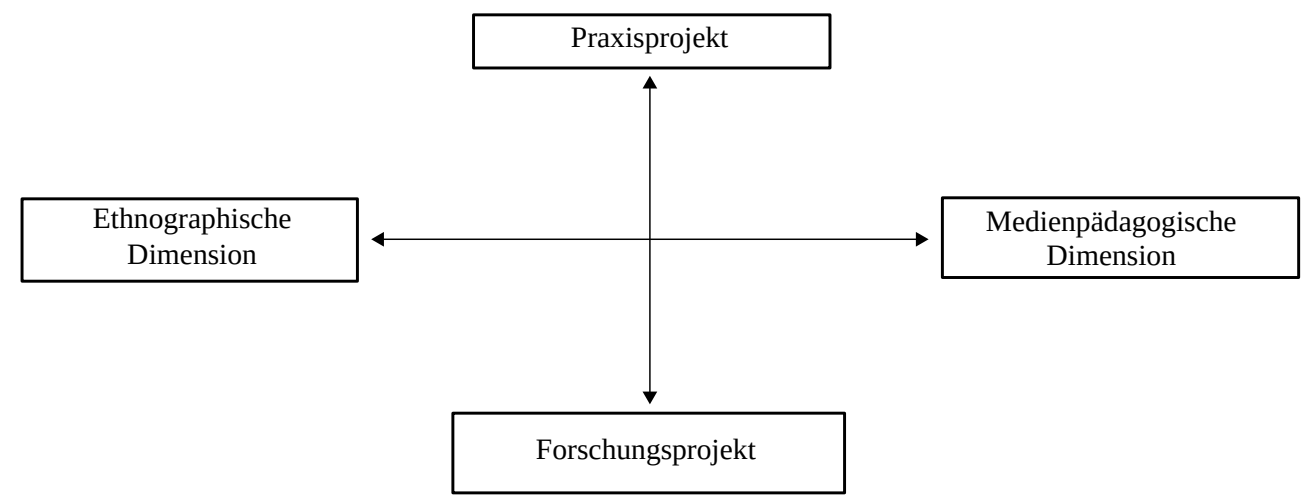

Abb. 3: CHICAM im Spannungsfeld verschiedener Forschungs- und Praxisdimensionen.

Das Praxisforschungsprojekt, das sich an der Tradition der Cultural Studies orientiert, ist im Schnittfeld von Migrationsforschung und medienpädagogischer Forschung angesiedelt und hat sowohl eine ethnographische als auch eine medienpädagogische Dimension. In Anlehnung an ethnographische Methoden werden die Lebenswelten der Kinder/ Jugendlichen erkundet. Auf der anderen Seite werden medienpädagogische Konzepte, die Reflexion und interkulturelle Kommunikation ermöglichen und vorhandene Kompetenzen aufgreifen und gezielt erweitern, angewendet und weiterentwickelt.

Das Projekt hat eine theoretische und eine praktische Dimension. Ziel des Projektes ist es, anschlussfähiges Wissen über die Situationen von Kindern aus Migrations- und Fluchtkontexten zu produzieren und über die Art und Weise, wie sie Medien nutzen können, um Erfahrungen zu reflektieren und auszutauschen. Indem Kinder die Möglichkeit bekommen, ihre Erfahrungen auszudrücken und mitzuteilen, kann erforscht werden, bis zu welchem Grad es möglich ist, ihre Stimmen und Bilder in unterschiedlichen öffentlichen Bereichen hörbar und sichtbar zu machen. Im Rahmen der praktischen Arbeit werden Medienkompetenzen vermittelt, die für die Kinder auch über die Projektarbeit hinaus nützlich sind.

7 Bisher (Ende 2001 bis Ende 2003) fanden insgesamt sechs internationale Projekttreffen sowie mehrere bilaterale Besuche statt. 


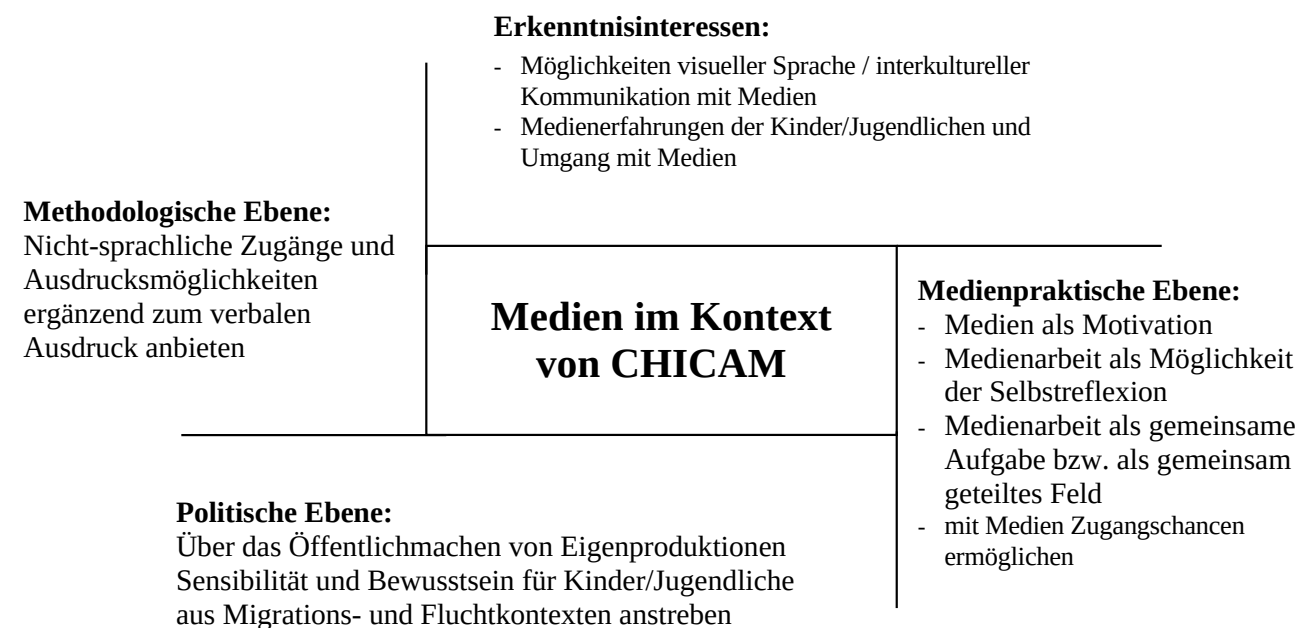

Abb. 4: Medienrelevante Ebenen bei CHICAM.

In dem oben stehenden Schaubild sind die verschiedenen Ebenen, in denen Medien bei CHICAM relevant sind, zusammengefasst (vgl. Abbildung 4).

\section{Stand der Projektarbeit}

Das Projekt befindet sich zurzeit im ersten Teil der Auswertungsphase (4). Forscherinnen und Forscher haben Beobachtungen Analysen und Fallstudien aus ihren jeweiligen Ländern zu den Themenkomplexen Peergroup, Schule und Familie erstellt. Die bilateralen Forscherteams, die jeweils für einen Themenkomplex zuständig sind, werden länderübergreifende thematische Analysen erstellen.

\subsection{Methoden und erste Erfahrungswerte}

Im Rahmen der Praxisforschung kamen folgende Methoden zum Einsatz:

- Teilnehmende Beobachtung und Begleitung der medienpraktischen Arbeit im CHICAM-Club. Audio-Aufnahmen ermöglichen eine spätere Vervollständigung der Feldnotizen.

- Teilnehmende Beobachtung und Begleitung der Kinder/Jugendlichen in verschiedenen lebensweltlichen Kontexten (z.B. Familienbesuche, Hospitationen im Klassenzimmer, Fußballverein, Computerspielen, Freibadbesuche).

- Interviews auf der Grundlage von Einwegfotos mit einzelnen Kindern/Jugendlichen und in 2er-Gruppen. Die Clubmitglieder wurden gebeten, zu bestimmten Themen (Tagesablauf im Alltag, Familie, Freunde, Schule, Besuch im Herkunftsland) Fotoaufnahmen mit Einwegkameras zu machen.

- Erstellung von kurzen Videoproduktionen unter medienpädagogischer Anleitung und Beratung (Knet-Animationsfilme, Spielfilme, reportageartige Befragungen). 
Die verschiedenen Phasen des Projekts

\section{1) Stand der Forschung (November 2001-Februar 2002)}

Sichtung und Reflexion des Forschungs- und Literaturstandes zu den Themen Kindheit Migration, und Medien (vgl. [http://www.chicam.net])

2) Pilotphase (Februar-Juli 2002)

Planung, Aufbau, Durchführung und Auswertung von CHICAM-Medienclubs in Griechenland, Italien, Großbritannien, Schweden, den Niederlanden und in Deutschland

\section{3) Hauptfeldphase (Juli 2002 bis Juli 2003)}

Planung, Aufbau und Durchführung von CHICAM-Medienclubs in Griechenland, Italien, Großbritannien, Schweden, den Niederlanden und in Deutschland

\begin{tabular}{|c|c|c|c|c|c|}
\hline \multicolumn{6}{|c|}{ 4) Auswertungsphase (Sommer 2003-Sommer 2004) } \\
\hline $\begin{array}{c}\text { Teilbericht zu } \\
\text { den }\end{array}$ & $\begin{array}{c}\text { Teilbericht zu } \\
\text { den }\end{array}$ & $\begin{array}{c}\text { Teilbericht zu } \\
\text { den }\end{array}$ & $\begin{array}{c}\text { Teilbericht zu } \\
\text { den }\end{array}$ & $\begin{array}{c}\text { Teilbericht zu } \\
\text { den }\end{array}$ & $\begin{array}{c}\text { Teilbericht zu } \\
\text { den }\end{array}$ \\
\hline $\begin{array}{c}\text { Workpackages 3, } \\
4 \text { und } 5 \text { aus } \\
\text { Griechenland }\end{array}$ & $\begin{array}{c}\text { Workpackages } 3 \text {, } \\
4 \text { und } 5 \text { aus } \\
\text { Italien }\end{array}$ & $\begin{array}{c}\text { Workpackages 3, } \\
4 \text { und } 5 \text { aus } \\
\text { Großbritannien }\end{array}$ & $\begin{array}{c}\text { Workpackages } 3 \text {, } \\
4 \text { und } 5 \text { aus } \\
\text { Schweden }\end{array}$ & $\begin{array}{c}\text { Workpackages } 3 \text {, } \\
4 \text { und } 5 \text { aus } \\
\text { den Niederlanden }\end{array}$ & $\begin{array}{c}\text { Workpackages } 3 \text {, } \\
4 \text { und } 5 \text { aus } \\
\text { Deutschland }\end{array}$ \\
\hline (Case studies) & (Case studies) & (Case studies) & & & (Case studies) \\
\hline$\downarrow$ & $\downarrow$ & $\downarrow$ & $\downarrow$ & $\downarrow$ & $\downarrow$ \\
\hline
\end{tabular}

Länderübergreifende Berichte zu den Workpackages 3, 4 und 5 (thematic analysis) (Themenkomplexe Peergroup, Schule und Familie)

\begin{tabular}{|c|c|c|c|c|c|}
\hline $\begin{array}{c}\text { Teilbericht zum } \\
\begin{array}{c}\text { Workpackage 6 } \\
\text { aus }\end{array}\end{array}$ & $\begin{array}{c}\text { Teilbericht zum } \\
\text { Workpackage 6 } \\
\text { aus } \\
\text { (Case studies) }\end{array}$ & $\begin{array}{c}\text { Teilbericht zum } \\
\text { Workpackage 6 } \\
\text { aus } \\
\text { Italien } \\
\text { (Case studies) }\end{array}$ & $\begin{array}{c}\text { Teilbericht zum } \\
\text { Workpackage 6 } \\
\text { (Case studies) } \\
\text { aus } \\
\text { Schweden } \\
\text { (Case studies) }\end{array}$ & $\begin{array}{c}\text { Teilbericht zum } \\
\text { Workpackage 6 } \\
\text { aus den } \\
\text { Niederlanden } \\
\text { (Case studies) }\end{array}$ & $\begin{array}{c}\text { Teilbericht zum } \\
\text { Workpackage 6 } \\
\text { aus } \\
\text { Deutschland } \\
\text { (Case studies) }\end{array}$ \\
\hline \\
Länderübergreifender Berichte zum Workpackage 6 (thematic analysis) \\
(Themenkomplex visuelle Kommunikation) \\
\hline \\
Gesamtbericht (summative analysis) \\
\hline
\end{tabular}

\section{5) ,Dissemination'-Phase}

Verbreitung der Ergebnisse (Projektpräsentationen, Tagungen, Publikationen, Erstellung von DVDs und Videos mit den Eigenproduktionen der Kinder/Jugendlichen) 


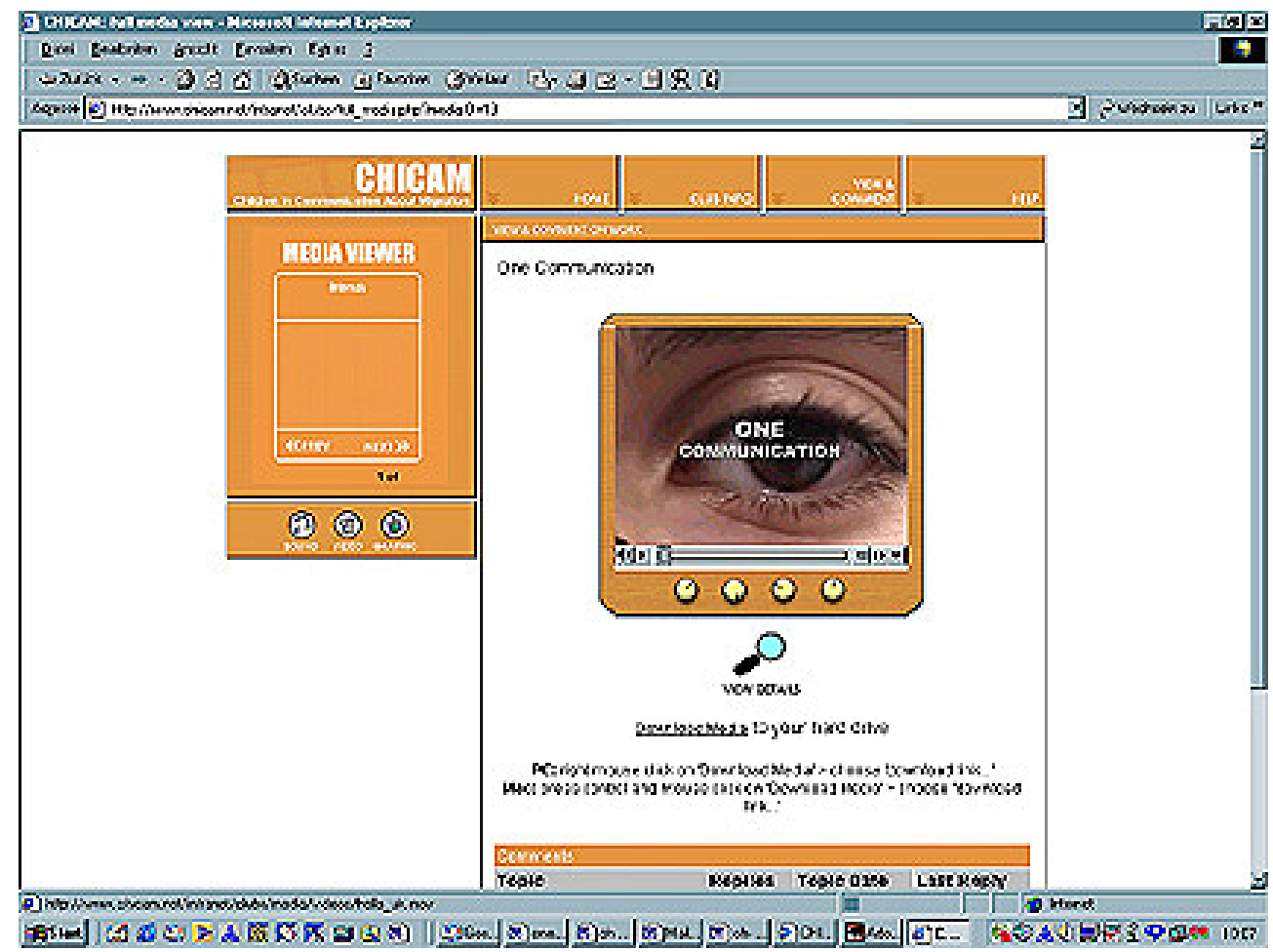

Abb. 5: Screenshot der CHICAM-Ciubmember-Intranetplattform.

- Dokumentation der Kommunikationsprozesse beim Austausch der Videoproduktionen über ein speziell eingerichtetes CHICAM-Clubmember-Intranet.

- Interviews zu Videoproduktionen. Die fertigen Videos wurden den ProduzentInnen gezeigt und sie konnten Aspekte, die ihnen wichtig waren, kommentieren.

Die entstandenen Eigenproduktionen und die Reaktionen anderer CHICAM-Clubs darauf werden ebenfalls ausgewertet. Dabei ist es wichtig, alle zur Verfügung stehenden Kontextinformationen zu berücksichtigen.

Die im Prozess entstehenden Eigenproduktionen haben für die verschiedenen am Projekt beteiligten Kinder/Jugendlichen und für verschiedene Projektdimensionen unterschiedliche Bedeutungen:

- Für die Kinder stellten die Produkte eine Vergegenständlichung ihrer Arbeit und ihres Könnens dar, etwas, das sie anderen zeigen können und worauf sie stolz sein können,

- die Produkte können einen Kommunikationsanreiz (z.T. auch Produktionsanreiz) für Partnergruppen in anderen Ländern darstellen, 
- die Produkte sind Präsentations- und Informationsmaterial in pädagogischen und politischen Kontexten, sie können auch Motivationsanreiz ${ }^{8}$ bedeuten,

- die Produkte dienen als Erzählstimulus für die Befragung („fotogeleitete Hervorlockung“( Harper 2000, S. 414)), - die Produkte werden zum Gegenstand von Analysen gemacht,

- die Produkte sind wichtig für Dokumentation, Illustration und Veranschaulichung (bei Projektpräsentationen und Publikationen).

Im Kontext von Forschung mit Kindern, die erst seit geraumer Zeit in Deutschland sind und eine andere Muttersprache sprechen, stellt sich das Problem der Sprache in zweifacher Weise: Wie kann sowohl der altersmäßigen Sprachentwicklung als auch den migrationsbezogenen Besonderheiten beim Spracherwerb Rechnung getragen werden?

Neuß gibt im Kontext von Interviews mit Kindern (ohne Migrationserfahrung) fünf nützliche methodische Hinweise (2000, S. 139f):

- Akzeptanz signalisieren: Die Lebenswelt des Kindes akzeptieren und respektieren;

- Einfache Fragen stellen: Es sollten nur solche Worte benutzt werden, die zum aktiven Wortschatz der Kinder gehören;

- Paraphrasieren: Den letzten Satz oder die letzten Worte des Kindes aufgreifen;

- Suggestivfragen vermeiden: Durch die Art des Fragens soll keine bestimmte Antwort nahegelegt werden;

- Bewertungen vermeiden: gegenüber den Aussagen eine (was Wertungen betrifft) neutrale Haltung einnehmen.

Der Interviewer muss sich an den Sprachkompetenzen der Interviewten orientieren, was jedoch nicht bedeuten kann, die Sprache auf Infinitive und Grundformen zu reduzieren („Du gehen Schule?“), zumal es bei CHICAM darum geht, den gesamten Forschungsund Praxisprozess auch als Vermittlung und Entwicklung von kommunikativen Kompetenzen zu sehen (medial und nichtmedial). Rydin und Westin (2002) weisen auf das Sprachproblem hin, das in vielen Forschungssituationen entstehen kann, wenn Forschende und Forschungssubjekte verschiedene Sprachgemeinschaften angehören. Sie unterscheiden die folgenden fünf Umgangsweisen, die jeweils mit verschiedenen Vorteilen und Nachteilen verbunden sind:

„1. The researcher learns to communicate in the minority language (the anthropological method).

2. The researcher limits his/her studies to minority groups whose language (s)he masters (selection of groups).

8 Bei einem praxisorientierten Workshop zum Thema interkulturelle Medienarbeit konnten wir die Erfahrung machen, dass Pädagoginnen und Pädagogen, die sich über Möglichkeiten der Etablierung von Medienarbeit in ihrer außerschulischen Jugendarbeit informieren wollten, durch Videobeispiele aus interkulturellen Projekten (u.a. VideoCulture und CHICAM) motiviert wurden. 
3. The researcher limits interviews to informants who speak the researcher's language (selection of individuals).

4. Interviews are conducted through interpreters (indirect access to respondent through intermediary).

5. Interviews are conducted by interviewers (other than the researcher) who speak the language. The taped interviews are subsequently transcribed and then translated (indirect access to respondent who is temporally removed)“ (Rydin/Westin 2002, S. 3).

Eine weitere Möglichkeit besteht darin, Subjekten, die sich - wie die Kinder und Jugendlichen im CHICAM-Club in Deutschland - im Prozess des Fremdspracherwerbs befinden, ergänzend zur Verbalsprache und diese unterstützend, visuelle Ausdrucksformen wie Fotografie und Film anzubieten (vgl. Teil 1).

Die ersten Erfahrungen haben gezeigt, dass ein Anknüpfen an selbstproduzierten Medienprodukten den Kindern das Sprechen über ihre eigene Lebenswelt erleichtert. Es deutet sich an, dass über Eigenproduktionen Zugänge zum

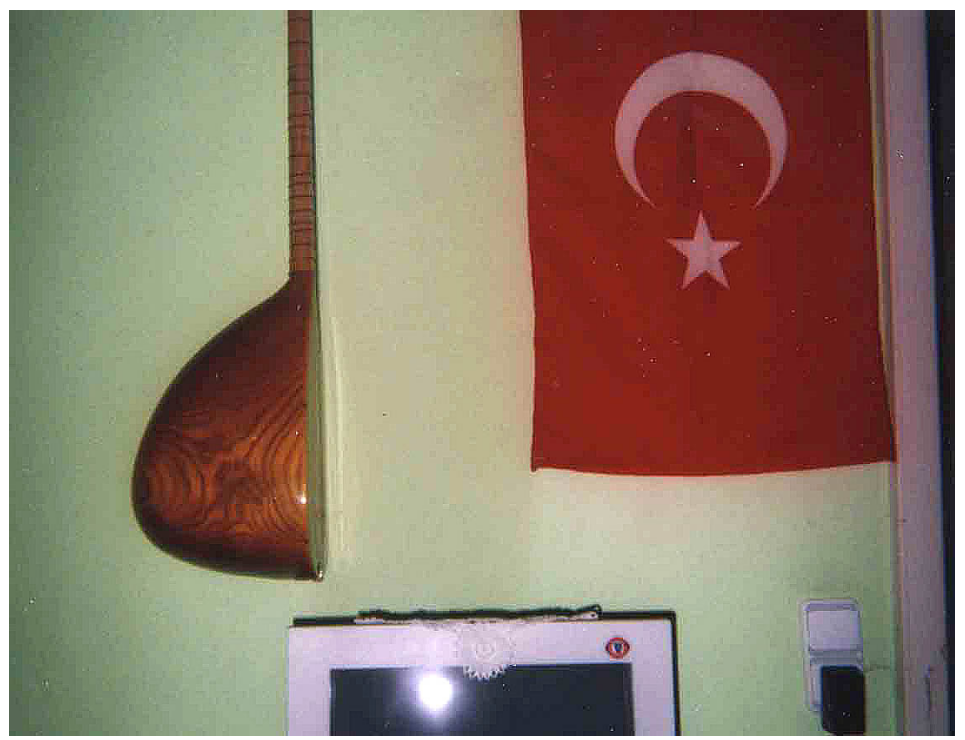

Abb. 6: Einwegkamerabild von Hakan: Saz (Saiteninstrument) und Türkische Flagge in der Wohnung?

9 Im Interview, das auf der Grundlage seiner Einwegfotobilder gemacht wurde, erzählt Hakan, dass er entgegen dem Wunsch seines Vaters nicht Saz lernen will:

Peter Holzwarth: Wie war des mit der Saz, kannst du?

Hakan: Hmm, mein Vater will, du gehst Saz-Kurse, dann sag ich „nein“.

Peter Holzwarth: Du willst nicht.

Hakan: Ja.

Peter Holzwarth: Und warum willst du nicht?

Hakan: Weil so schwer. 


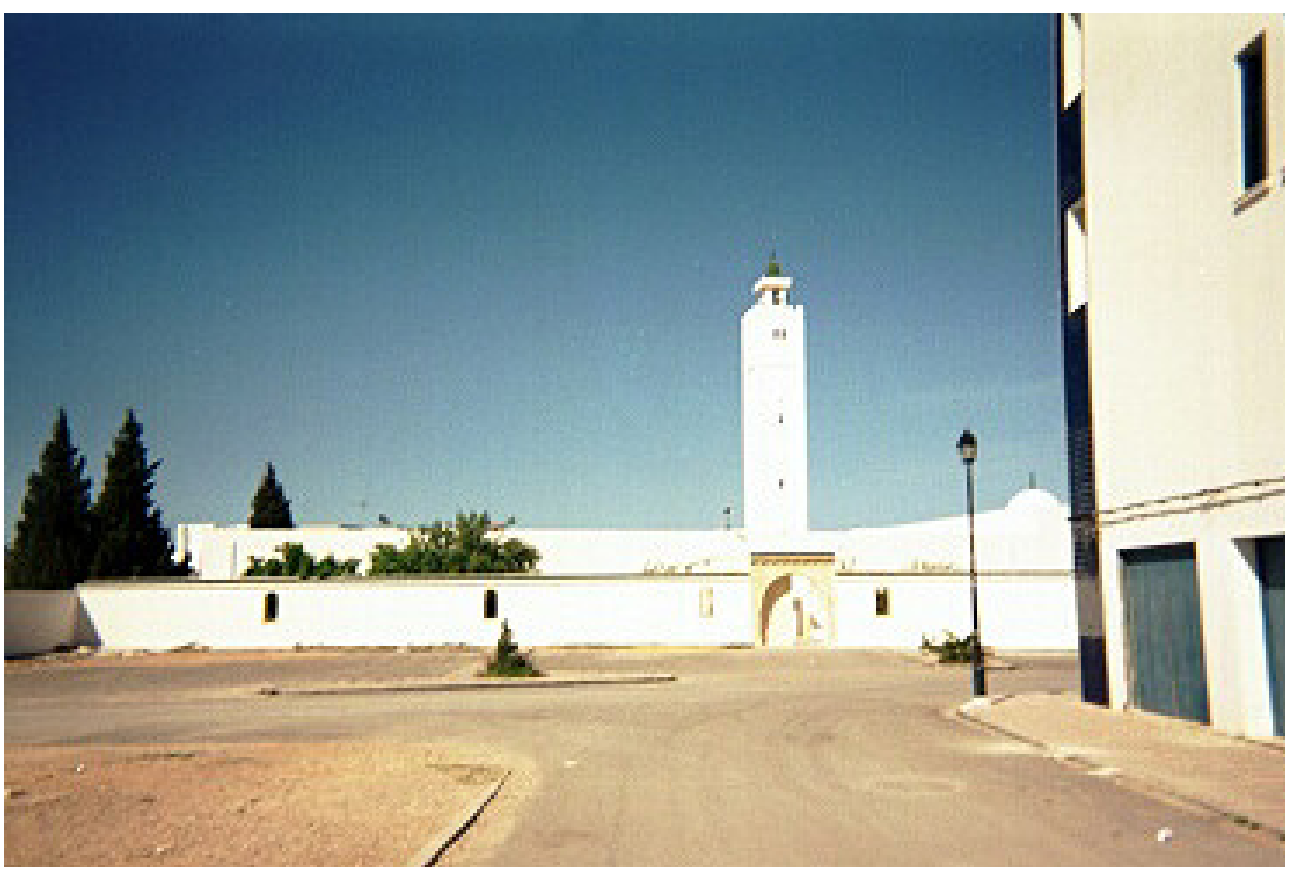

Abb. 7: Einwegkamerabild von Mustafa: Moschee in Tunesien.

Welterleben der Kinder eröffnet werden können, die ansonsten aufgrund von Kommunikationsbarrieren versperrt bleiben würden. Indem den Kindern z.B. Einwegfotoapparate mitgegeben werden, eröffnen sich neue Zugänge in Bezug auf das Erleben von Migration. Dies gilt sowohl für fotografische Annäherungen an lebensweltliche Umgebungen in Deutschland als auch für etwaige Besuche im Herkunftsland (vgl. Abbildungen 6 und 7).

Die Verbindung von Forschung und praktischer Medienarbeit trägt dazu bei, dass der Forschungsprozess durch einen Charakter des gegenseitigen Gebens und Nehmens geprägt wird. Die am Projekt beteiligten Kinder geben Einblicke in ihre Lebenswelt, die beteiligten Erwachsenen vermitteln Medienkompetenz, ermöglichen Spaß und neue Erfahrungen und sie geben Aufmerksamkeit. Neben Lernerfahrungen und sozialen Erfahrungen stellen die entstandenen Produktionen etwas konkret Gegenständliches dar, das die Kinder aus dem Projekt mitnehmen können. Ein weiterer Vorzug des Ansatzes besteht darin, dass sich über die Medienarbeit verschiedene informelle Gesprächssituationen ergeben, die eine Erhebung von Daten außerhalb offiziell als Interview gerahmter Settings ermöglichen.

Insgesamt waren bei den Kindern/Jugendlichen Produktionen attraktiver, die sich innerhalb kürzerer Zeit fertig stellen lassen und wenig Frustrationstoleranz erfordern. Schnelle Rückmeldung auf das fertige Produkt war also ein sehr wichtiges Kriterium. Produktionsformen, die Zeit und Geduld erfordern und mit Auswählen und Schneiden von Filmmaterial verbunden sind, waren für viele der Clubmitglieder weniger motivierend. Zum Teil schien es ihnen auch gar nicht so sehr um ein fertiges Produkt zu gegen, sondern um den Spaß im 
Entstehensprozess. Neben Knetanimationen (die keiner Nachproduktion bedürfen) und kleinen narrativen Filmen war bei den Kindern/Jugendlichen eine reportageartige Form beliebt, bei der sie mit der Videokamera auf die Straße gehen und Passanten befragen konnten. Im Rahmen des ersten Projekts „Deutschland positiv/negativ“10 wurden Leute gefragt was ihnen an Deutschland gefällt und was ihnen nicht gefällt. Im zweiten Projekt ging es um Stimmen und Meinungen zum Golfkrieg. Die Kinder/Jugendlichen hatten im Club selbst das Bedürfnis geäußert, zu diesem Thema eine Befragung zu machen. Bei diesem Beispiel handelt es sich um einen Entwicklungsprozess, bei dem es nicht nur um einen Zuwachs an Medienkompetenz geht, sondern auch um soziale und kommunikative Dimensionen (Selbstbewusstsein, Selbstüberwindung).

Sowohl Susan (13 Jahre, aus den USA) als auch Taskania (14 Jahre, aus der Dominikanischen Republik) machten die Erfahrung, dass sie gegenüber den ersten Kamerabefragungen auf der Straße beim zweiten Mal weniger ängstlich und mit mehr Mut und Selbstsicherheit auf fremde Menschen zugehen konnten:

Susan: $\quad$ Und ich finde auch dass ähm finde das auch jetzt gut aber früher nich mehr also wann ma so rumgelaufen sind im Stadt und so gefragt und so find ich gut jetzt

Peter Holzwarth: Und früher nich so?

Susan: $\quad$ Früher nich so da war ich da hat ich noch mehr Angst

Peter Holzwarth: Früher heißt bei dem bei dem ersten Film, wo ihr zum ersten Mal Leute angesprochen habt, mhm Susan: Aber jetzt nich mehr.

Peter Holzwarth: $\mathrm{mhm}$

Susan: $\quad$ Also auf letzten hab ich jede gefragt, Taskania hat auch ge...

Peter Holzwarth: Und wie kam des, dass des sich so verändert hat? Also erst so ängstlich und jetzt ganz mutig?

Susan: $\quad$ Vielleicht weil des des zweite mal war. Oder dritte mal so.

Ähnlich äußert sich auch Taskania:

Peter Holzwarth: Und hast du n Unterschied gemerkt beim zweiten mal, wo's um den Irak-Krieg ging und ähm zum ersten mal wo’s um Deutschland ging, was den Leuten gefällt und nicht gefällt. Also gab’s n Unterschied zwischen dem ersten mal wo’s um Deutschland ging und dem zweiten mal wo's um den Irak-Krieg ging?

Taskania: (Lachen)

Björn Maurer: $\quad$ Wo du das erste Mal auf Leute zugegangen bist mit der Kamera.

Taskania: Ja habe ich, habe ich ein bisschen Angst gehabt, aber zweites Mal nicht mehr.

Björn Maurer: Und als wir dann noch mal losgezogen sind oder als ihr noch mal losgezogen seid mit der Kamera zum Irak? Des war ja dann ...

Taskania: Ja, keine Ahnung mehr haben wir gehabt. Des war so. Hat bei uns Spaß gemacht.

10 Eine Transkription des Videos befindet sich im Anhang. 


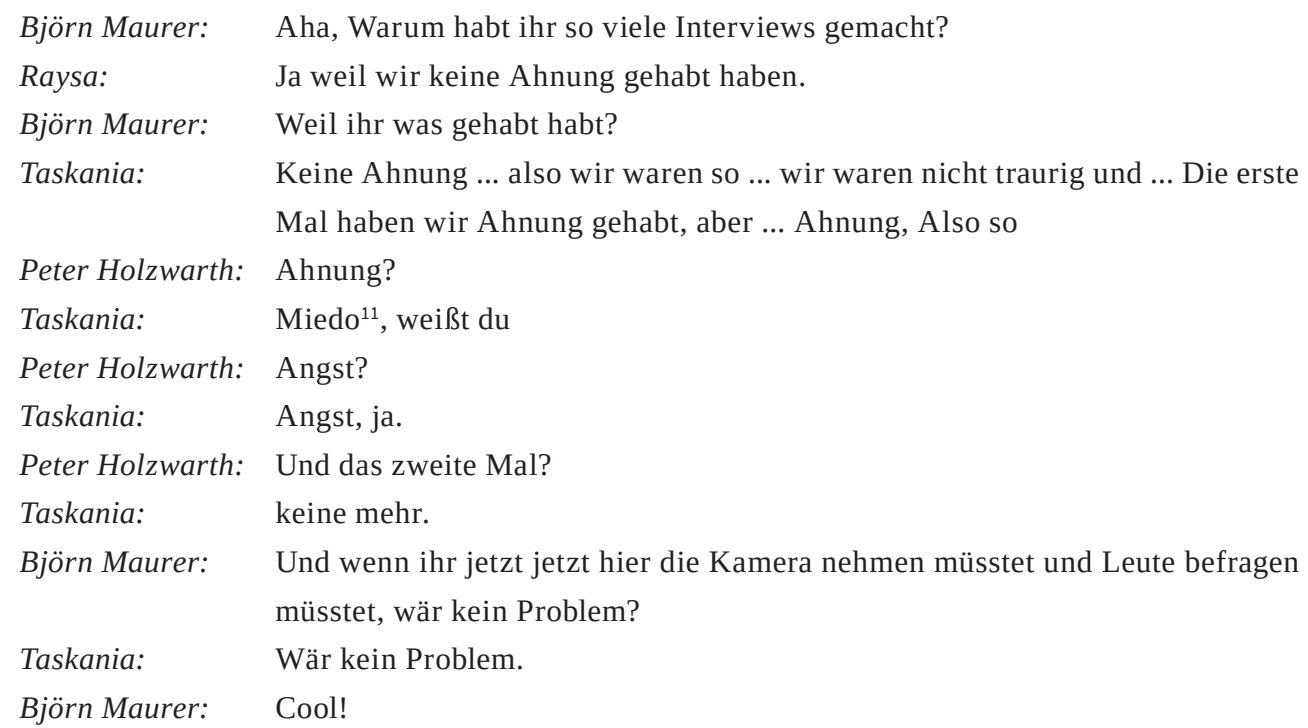

Dieses Beispiel verdeutlicht, dass in Kontexten, in denen sich die Sprachkompetenzen von Interviewer und Befragten nur zum Teil überschneiden, gegenseitiges Verstehen an Grenzen stoßen kann und dass es für Forschende von großem Vorteil ist, die Muttersprache des Interviewpartners zu kennen.

An der genannten Produktion „Deutschland positiv/negativ“ lässt sich zeigen, wie durch aktive Medienproduktion Reflexion und Kommunikation ausgelöst werden kann und sich dadurch die Forschenden wichtige Themenbereiche eröffnen. Bei der Sichtung der Aufnahmen zu diesem Film wurde die Aussage eines Mädchens mit Migrationshintergrund, die Lehrer seinen streng in Deutschland, zum Ausgangspunkt einer engagiert geführten Gruppendiskussion über Freiheit, Disziplinierungsmöglichkeiten und körperliche Bestrafung. Über die Reflexion des gefilmten Materials wurde unter anderem deutlich, dass das Thema Kopftuchtragen, die mit dem Kopftuch verbundenen Machtverhältnisse im Generationenverhältnis und die Beurteilung des Kopftuchs durch Mitschüler und Lehrer für einige der Clubmitglieder von zentraler Bedeutung war.

Im Zusammenhang mit Lernfortschritten im Spracherwerb kam ein Lehrer der Kinder/Jugendlichen zu folgender Einschätzung in Bezug auf CHICAM:

„Das ganze war 'ne Belebung für die Klasse für die Gruppe auch und hat hineingewirkt in die Klasse hinein auch ... des isch was Außergewöhnliches gewese’ und kommt auch nicht wieder so schnell.“ [...]

„Ich glaube auch gerade durch des CHICAM muss ich ehrlich sagen, ja, des hat des is, war 'ne sehr gute Sache, die wirklich motiviert hat und die den Schülern auch geholfen hat gerade mit ihnen zusammen dann in der Gruppe etwas zu erleben und dabei auch zu gewinnen an Sprachwert, an Sprachqualität.“

11 miedo (span.): Angst. 
Die Klassenlehrerin ergänzte: „Weil der CHICAM-Club der setzt sich ja doch aus verschiedenen Nationen zusammen, da müssen sie einfach Deutsch reden, ja, und des geht dann automatisch.“

Diese Aussagen sowie die Beobachtungen im CHICAM-Club über ein Jahr hinweg machen deutlich, dass die Projektpraxis einen wichtigen Beitrag zur Sprachentwicklung leisten konnte, auch wenn dieser Aspekt gar nicht explizit Teil der Intentionen war. Sprachliche Fortschritte konnten sich wiederum positiv auf die Verstehensmöglichkeiten von medienpädagogischen Inputs auswirken, was die Aneignung von Medienkompetenzen erleichtert. Möglicherweise ist gerade der Umgang mit visuellen und audiovisuellen Medien, an dem die wortsprachliche Kommunikation anknüpfen kann, besonders zur Sprachentwicklung geeignet. Dieser Aspekt soll bei der weiteren Auswertung beachtet werden.

\section{Literatur}

Baacke, D.: Kevin, Wayne und andere: Kinder und ästhetische Erfahrung. In: von Gottberg, J./Mikos, L./Wiedemann, D. (Hrsg.): Kinder an die Fernbedienung. Berlin 1997, S. 13-31.

Belgrad, J./Niesyto, H: Symbol. Produktion und Verstehen in pädagogischen Kontexten. Hohengehren 2001a.

Belgrad, J./Niesyto, H: Symbolverstehen und Symbolproduktion. In: Belgrad, J./Niesyto, H. (Hrsg.): Symbol. Verstehen und Produktion in pädagogischen Kontexten. Hohengehren 2001b, S. 5-16.

Bourdieu, P: Die feinen Unterschiede. Kritik der gesellschaftlichen Urteilskraft. Frankfurt am Main 1987.

Buckingham, D.: Children and Media Overview. In: Global Kids, Global Media: a review of research relating to children, media and migration in Europe. Research project „CHICAM-Children in Communication about Migration“, 2002, S. 99-115. [http://www.chicam.net/research.html].

Charlton, M./Neumann-Braun, K: Medienkindheit, Medienjugend. München 1992.

Ehrenspeck, Y/Schaffer, B. (Hrsg.): Film- und Fotoanalyse in der Erziehungswissenschaft. Ein Handbuch. Opladen 2003.

Fix, M./Melenk, H.: Schreiben zu Texten. Schreiben zu Bildimpulsen. Das Ludwigsburger Aufsatzkorpus. Hohengehren 2000.

Friedrich, M. u.a. (Hrsg.): Die Fremden sehen. Ethnologie und Film. München 1984.

Fuhs, B.: Fotografie als Dokument qualitativer Forschung. In: Ehrenspeck, Yvonne/Schäffer, B. (Hrsg.): Film- und Fotoanalyse in der Erziehungswissenschaft. Ein Handbuch. Opladen 2003, S. 37-54.

Fuhs, B.: Fotografie und qualitative Forschung. Zur Verwendung fotografischer Quellen in den Erziehungswissenschaften. In: Friebertshäuster, B./Prengel, A. (Hrsg.): Handbuch qualitative Methoden in der Erziehungswissenschaft. Weinheim/München 1997, S. 265-285.

Harper, D.: Fotografien als sozialwissenschaftliche Daten. In: Flick, U./von Kardorff, E./Steinke, I. (Hrsg.): Qualitative Forschung. Ein Handbuch. Reinbek bei Hamburg 2000, S. 402-416.

Hepp, A.: Cultural Studies und Medienanalyse. Opladen/Wiesbaden 1999.

Hepp, A./Winter, R. (Hrsg.): Kultur - Medien - Macht. Cultural Studies und Medienanalyse. 2. Auflage. Opladen/Wiesbaden 1999.

Hiegemann, S./Swoboda, W. H: Handbuch der Medienpädagogik. Opladen 1994.

Holzwarth, P: Graffitikultur als Gegenstand der Jugendforschung. In: Niesyto, H. (Hrsg.): Selbstausdruck mit Medien. Eigenproduktionen mit Medien als Gegenstand der Kindheits- und Jugendforschung. München 2001, S. 79-88.

Holzwarth, P: Children and Migration: Germany. In: Global Kids, Global Media: a review of research relating to children, media and migration in Europe. Research project „CHICAM - Children in Communication about Migration“, 2002, S. 19-33. [http://www.chicam.net/research.html]. 
Holzwarth, P./Maurer, B.: Kreative Bedeutungskonstruktion und ästhetische Reflexivität im Spannungsfeld von Symbolproduktion und Symbolverstehen. Eine fallbezogenen Analyse. In: Niesyto, H. (Hrsg.): VideoCulture. Video und interkulturelle Kommunikation. München 2003, S. 139-168.

Holzwarth, P./Maurer, B.: Children and Media: Germany. In: Global Kids, Global Media: a review of research relating to children, media and migration in Europe. Research project „CHICAM - Children in Communication about Migration“, 2002, S. 117-131. [http://www.chicam.net/research.html].

Holzwarth, P/Maurer, B./Niesyto, H: CHICAM - CHILDREN IN COMMUNICATION ABOUT MIGRATION. In: Gesellschaft für Medienpädagogik und Kommunikationskultur e.V.: nexum das Netzwerk. Heft 8/2002, 2002, S. 19-21.

Jugendwerk der Deutschen Shell (Hrsg.): 11. Shell-Jugendstudie. Jugend '92: Lebenslagen, Orientierungen und Entwicklungsperspektiven im vereinigten Deutschland. Band 1. Opladen 1992.

Langer, S. K, (1942): Philosophie auf neuem Wege. (Cambridge/Mass.) Frankfurt/M. 1987. Maurer, B.: Wenn Jugendliche mediale Wirklichkeit erzeugen ... Konzeptionelle und praktische Überlegungen zur handlungsorientierten Videoarbeit. In: Belgrad, Jürgen/Niesyto, H (Hrsg.): Symbol. Verstehen und Produktion in pädagogischen Kontexten. Hohengehren 2001a, S. 104-114.

Maurer, B.: Formen der medienpädagogischen Begleitung im Kontext von Jugendforschung mit Video. In: Niesyto, H. (Hrsg.): Selbstausdruck mit Medien. Eigenproduktionen mit Medien als Gegenstand der Kindheits- und Jugendforschung. München 2001b, S. 173-185.

Mayring, Ph./Gläser-Zikuda, M./Hurst, A.: Qualitative Ansätze der Erforschung von Eigenproduktionen mit Medien am Beispiel von Tagebuchanalysen und Fallanalysen. In: Niesyto, H. (Hrsg.): Selbstausdruck mit Medien. Eigenproduktionen mit Medien als Gegenstand der Kindheitsund Jugendforschung. München 2001, S. 15-33.

Neuß, $N$ : Medienbezogene Kinderzeichnungen als Instrument der qualitativen Rezeptionsforschung. In: Paus-Haase, I./Schorb, B.: Qualitative Kinder- und Jugendmedienforschung. München 2000, S. 131-154.

Neuß, $N$ : Symbolische Verarbeitung von Fernseherlebnissen in Kinderzeichnungen. Eine empirische Studie mit Vorschulkindern. München 1999.

Niesyto, H: Erfahrungsproduktion mit Medien. Selbstbilder, Darstellungsformen, Gruppenprozesse. Weinheim/München 1991.

Niesyto, H: Sozialvideografie. Mediale Exploration als spezifischer Zugang zur ästhetisch-symbolischen Dimension jugendkultureller Milieus. In: Institut für Sozialpädagogik und Sozialarbeit der TU Dresden/Institut für regionale Innovation und Sozialforschung IRIS e.V (Hrsg.). Dresden 1996: Hochschulschrift, S. 66-95.

Niesyto, H: Video als Ausdrucksmedium. Zur medienethnografischen Exploration jugendkultureller Symbolmilieus. In: Fromme, J. u.a. (Hrsg.): Selbstsozialisation, Kinderkultur und Mediennutzung. Opladen 1999, S. 327-344.

Niesyto, H: Youth Research on Video Self-productions. Reflections on a Social-aesthetic Approach. In: Visual Sociology 15 (2000), 2000a, S. 135-153.

Niesyto, H: Medienpädagogik und soziokulturelle Unterschiede. Eine Studie zur Förderung der aktiven Medienarbeit mit Kindern und Jugendlichen aus bildungsmäßig und sozial benachteiligten Verhältnissen. Baden-Baden/Ludwigsburg 2000b: Medienpädagogischer Forschungsverbund Südwest/PH Ludwigsburg.

Niesyto, H. (Hrsg.): Selbstausdruck mit Medien. Eigenproduktionen mit Medien als Gegenstand der Kindheits- und Jugendforschung. München 2001a.

Niesyto, H.: Jugendforschung mit Video. Formen, Projekte und Perspektiven eines Forschungsansatzes. In: Niesyto, H. (Hrsg.): Selbstausdruck mit Medien. Eigenproduktionen mit Medien als Gegenstand der Kindheits- und Jugendforschung. München 2001b, S. 89-102.

Niesyto, H: Qualitative Jugendforschung und symbolischer Selbstausdruck. In: Belgrad, J./Niesyto, H (Hrsg.): Symbol. Verstehen und Produktion in pädagogischen Kontexten. Hohengehren

Niesyto, H. (Hrsg.): VideoCulture. Video und interkulturelle Kommunikation. Grundlagen, Methoden und Ergebnisse eines internationalen Forschungsprojekts: München 2003.

Paus-Haase, I: Medienrezeptionsforschung mit Kindern - Prämissen und Vorgehensweisen. Das Modell der Triangulation. In: Paus-Haase, I./Schorb, B.: Qualitative Kinder- und Jugendmedienforschung. München 2000, S. 15-32.

Prosser, J./Schwartz, D.I Photographs within the Sociological Process. In: Prosser, Jon (Hrsg.): Imagebased Research. A Sourcebook for Qualitative Researchers. London 1998, S. 115-130. 
Rydin, I./Westin, Ch.-. Notes on Interviewing. CHICAM methodology discussion paper. Unveröffentlichtes Projektdokument 2002.

Schorb, B./Theunert, H.: Kontextuelles Verstehen der Medienaneignung. In: Paus-Haase, I./Schorb, B.: Qualitative Kinder- und Jugendmedienforschung. München 2000, S. 33-57.

Schmid, A.: Mediale Eigenproduktionen Jugendlicher zwischen Selbst- und Fremdbestimmung in professionstheoretischer Perspektive - eine ethnographisch-gesprächsanalytische Fallstudie. In: Niesyto, $H$. (Hrsg.): Selbstausdruck mit Medien. Eigenproduktionen mit Medien als Gegenstand der Kindheits- und Jugendforschung. München 2001, S. 53-66.

Theunert, $H$ : Quantitative versus qualitative Medien- und Kommunikationsforschung? Uber Grundsätze, Gegensätze und Notwendigkeiten der Ergänzung heutiger methodologischer Paradigmen. In: Hiegemann, S./Swoboda, W. H. (Hrsg.): Handbuch der Medienpädagogik. Opladen 1994, S. 387-401.

Vogelgesang, W.: Jugendliche Video-Cliquen. Action- und Horrorvideos als Kristallisationspunkte einer neuen Fankultur. Opladen 1991.

Westin, Ch.: Children and Migration Overview. In: Global Kids, Global Media: a review of research relating to children, media and migration in Europe. Research project „CHICAM - Children in Communication about Migration“, 2002, S. 7-17. [http://www. chicam.net/research.html].

Winterhager-Schmid, L.: Jugendtagebuchforschung. In: Friebertshduster, Barbara/Prengel, Annedore (Hrsg.): Handbuch qualitative Methoden in der Erziehungswissenschaft. Weinheim/München 1997, S. 354-370.

Witzke, M.: Identität, Selbstausdruck und Jugendkultur. Eigenproduzierte Videos Jugendlicher im Vergleich mit ihren Selbstaussagen. Ein Beitrag zur Jugend(kultur)forschung. München 2003.

\section{Anhang - Transkription des Videos „Deutschland positiv/negativ“ \\ + positiv}

Junger Mann: Besonders gut? Die Autobahnen.

Susan: Warum?

Junger Mann: Warum? Weil man schnell fahren darf.

\section{- negativ}

Junge Frau mit Migrationshintergrund: Die Leute sind kalt.

Björn: Ja.

Junge Frau mit Migrationshintergrund: Sie wollen keinen Kontakt haben.

\section{+ positiv}

Hakan: Äh, Deutschland ist sehr modern. Schule ist auch sehr gut. Äh, in die Schule kann nicht schlagen beim Lehrer. Und so alles, gut.

\section{- negativ}

Serife: Die Sommer gefällt mir nicht, weil es gibt net so viel Sonne. Und die und die Landratsamt gefällt mir nicht. 


\section{+ positiv}

Susan: Oh toll (Missfallensäußerung wegen der bisher unbefriedigenden Antworten)

Gruppe von männlichen Teenagern mit Migrationshintergrund: Ja ok die Menschen hier, die Menschen gefallen, die Freundlichkeit, Menschen, die Leben, die Freundlichkeit, die Frauen.

\section{- negativ}

Älterer Mann: Ja manchmal die Kriminalität.

\section{+ positiv}

Susan: Wenn's Schnee gibt gefällt mir ... (unverständlich) und dass wir können Busfahren wann wir wollen.

- negativ

Meral: (spricht auf Türkisch)

Serife übersetzt: Die Kultur gefällt nicht.

\section{+ positiv}

Frau mit Migrationshintergrund (mit Kopftuch): Deutschland was gefällt mir, mhm das System, Disziplin ...

Susan: Mhm

Frau mit Migrationshintergrund (mit Kopftuch): ... die Ordnung

Hakan: Was gefällt in ihnen in Deutschland?

Mann mit Berliner Akzent: In Deutschland?

Hakan: Ja.

Mann mit Berliner Akzent: Ja alles mein Gott das Leben ist so schön oder, solange kein Krieg ist gell und wir alle zu essen und zu trinken haben n Dach überm Kopf und und sie gehen ja noch zur Schule gell.

Hakan: Ja.

Mann mit Berliner Akzent: Sie können zur Schule gehen nicht überall kann man zur Schule gehen gell, das is ne tolle Sache. Da hab ich viel gelernt.

\section{- negativ}

Frau: Der Euro (lachen) Der Euro.

Susan: War's besser mit äh D-Mark?

Frau: D-Mark war viel besser.

Susan: OK.

Schülerin (möglicherweise mit Migrationshintergrund): Die Lehrer sind ganz schön streng.

Mädchen mit Migrationshintergrund: Ich weiß net also ich kann des jetzt nur mit der Türkei vergleichen, weil in der Türkei kann man net so frei alleine als Mädchen rumlaufen und hier hat man seine Ruhe vor den Leuten. Da stresst dich halt net so viele. 